it for that of his later namesake, whose denomination appears in various forms and in only one manuscript is given as Gillitanus. ${ }^{17}$ If he came from Gillium, he wrote at a date earlier than the Arab invasion, and it would not be easy to show how his cycle travelled into western Europe. If on the other hand, as I have suggested, he belonged to Squillace, the transmission of his manuscript would be readily intelligible.

Regratd L. Poole.

\title{
Cardinal Ottoboni and the Monastery of Stratford Langthorne
}

Wring Ottoboni, cardinal deacon of St. Adrian, was in England as legate from 1265 to 1268 , he exercised his power of visiting exempt monasteries and Orders. ${ }^{1}$ But he met with resistance from the Abbot and Convent of Stratford Langthorne, an important Cistercian monsstery in Esser, a few miles from London. They refused to admit two Franciscans who were sent by Ottoboni to visit them, ${ }^{2}$ and appealed to Clement IV, in virtue of pepal privileges which had been granted to the Cistercian Order. I have been unable to find any other reference to this dispute, so that it is impossible to discover if the abbot and convent finally submitted to the legate's visitation. The series of documents concludes with a humble letter from the abbot to the cardinal on behalf of two monks who had evidently been punished by him, and forbidden to exercise their functions as priests. When Ottoboni was besieged in the Tower of London by the earl of Gloucester in 1267 he was released by Henry III, who brought him to the monastery of Stratford. Peace was made there with the barons on 6 June $1267 .^{3}$

The proceedings printed below are found in MS. 499, ff. $257^{\mathrm{V}}$ 261, in the Lambeth Palace library ; it is a quarto of 345 folios, written in a minute and much contracted hand, probably in the early years of the fourteenth century. The contents are miscellaneous, and include several works of St. Angustine." From f. 252 onwards there are records and proceedings relsting to Cistercian monasteries, forms, letters, and charters, e.g. letters from Robert Grossetete, bishop of Lincoln, to the papal curia,

"MS. 298 of St. Romi at Rheims, eoconding to Jenms, Bist. Cydi Dtonysiani (Wittenberg, 1718), p. 51.

1 o. g. Westmingter (Cotton MS., Fantine A. iii, f. 210), and the Order of Sempringham (Douce MS. 136, 1 88, Bodloien Library).

- Clement IV gave Ottoboni the powrer of compelling any of the triars to undertake sny commission for him: Bullariwn Pranciocanxm, ed J. H. Sbaralen, iii. 9. no. 12.

1 Anxales Monastici, ed. Luerd, ii. 105; iv. 201, 208, 205.

- See Todd, Catalogue of IISS. in the Libonry of Lambeth Palace, P. 64. 
the reissue of Magna Carta in 1217, and the Charter of the Forest. No dated document appears to be later than 1274.

Some charters concern the Cistercian monastery of $\mathrm{L}$. in the diocese of Coventry and Lichfield, which I have identified with Stanlaw in Cheshire, often described in charters as Locus benedictus de Stanlawe. ${ }^{5}$ The monks of Stanlaw were transferred to Whalley in 1296, and as the word Whalley is written inside the cover of MS. 499 there can be little doubt that it was formerly in the library of that monastery. Stanlaw was probably founded by monks from Combermere, ${ }^{6}$ which, like Stratford Langthorne, was among the English houses of the Order of Savigny ; they were united to Citeaux in 1147 , but were reckoned as daughter houses of Savigny. This connexion explains how a record of proceedings concerning Stratford Langthorne comes to be found in a manuscript at Whalley. ROSE GrarAM.

Inmbeth 198. 498, fo. 202.

1. $967^{\circ}$

1. 248 .

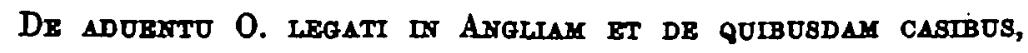
PBOCESSIBUS, BT LITTHRIS IPSUX BT ALIOS TANGEATIBU8.

De adventer 0 . logati in Angliam anno $\mathrm{M}^{\circ} \cdot \mathrm{C} \mathrm{C}^{0} \cdot L X V .{ }^{\mathrm{B}}$

Memorandum quod anno domini Ma.CCo.LXV v venit Othobonus apostolice sedis legatus in Angliam deferens secum litteras Clementis pape qui sedit ante Gregorium decimum ${ }^{10}$ in hec uerba :-Clemens episcopus seruns sernorum dei dilecto filio Othobono sancti Adriani discono cardinsli spostolice sedis legato salutem et apostolicam benedictionem. Cum te ad partes Anglie et commisso inibi ac in regno Scocio Wallie et Hybernie plene legacionis officio pro urgenti et arduo negocio destinemns, quis in desideriis nostris grauiter ut commissum tibi negocium amotis impedimentis quibuslibet felicem consequstur effectom, priusndi quoslibet religiosos cuinscumque ordinis, qui super hiis que spectant ad tue legacionis officiom et aliis tibi commissis a te moniti plensrie tibi parere contempserint, omnibus indulgenciis et prinilegïis eis ab apostolics sede concessis, discrecioni tue plensm concedimus anctoritate presencinm facultatem. Detum Perusii iij nonis Maii pontificstus nostri anno primo.

Clemens episcopus et cetera, sicut andiuimus, nonnullis religiosis tue legacionis scilicet Cluniscensium et aliorum ordinum a sede apostolica sit indultam quod legati einsdem sedis eos absque speciali mandato sedis einsdem faciente plenam et expressam de indulto huinsmodi mencionem

The charters on ff. 262, 263 are printed in the Coucher Bool of Whalley, od. Holton (Chotham Socioty), ii. 425, 428.

- Ibid. $\mathrm{z}$ iv.

Trte, viii. 669, 675.

- MS. $\mathrm{H}^{\circ} \mathrm{CC} \cdot \mathrm{LXX}$ Cardinal Ottoboni arrived in England on 29 Octobor 1265, and left this comtry on 28 July 1288 : Arates Momastici, iv. 218.

Ibid.

10 The acribe almost invariebly indicates numerale by puxeling aigns, which I hsro dociphered through bis use of them in nambering the titles of the cheptern of the Bools of the Decretale on ff. 252\%, 252 . I heve cince found them reproduced with their Romen equiralents in Mather Peris' Chrovica maiora, $\nabla .985$, where it is aid that they were brought to England by John of Besingroke, who had stadied at Athens. 
nequeant visitare, nos volentes quod aliqui a tua visitacione pretextu indulti huinsmodi se tueri non valeant, discrecioni tue vt tales quouis indulto huiusmodi sedis apostolice non obstante uisitare ualeas tibi auctoritate presencium concedimus facultatem. Datum Perusii ot supra.

\section{Aliud procuratorium.}

Clemens episcopus et cetera. II Cum prosperum regni Anglie statum plenis desideriis affectantes te de cuins industria et circumspeccione confidimus ad idem regnum commisso tibi tam inibi quam in quibusdam aliis partibus plene legacionis officio de fratrum nostroram consilio pro reformacione status eiusdem regni durimus destinandum. Vt autem in commisso tibi huiusmodi officio deo propicio uel propiciante valeas prosperari, exercendi libere per te uel per alium nel alios censuram ecclesiasticam in venerabiles patres archiepiscopos nostros et episcopos; ac in cathedralium et aliarum ecclesiarum domorum et monasteriorum tam exemptorum quem non exemptorum prelatos et clericos conuentus st capitula, necnon comites barones et nobiles potestates rectores balliuos consilis communis vinversitates et populos locornm cuinslibet legacionis tue, et quascomque personas ecclesiasticas et seculares publicas et prinatas cuiuscumque ordinis condicionis sen dignitatis existant et terras eoram eiusdem legacionis tue cum videris expedire, non obstantibus aliquibus privileguis uel indulgenciis quibuscumque personis locis seu ardioibus sub quanis uerborum forma, ab apostolics sede concessis de quibus quoramque tenoribus plenam et expressam ac de nerbo ad uerbum opporteat in nostris litteris fieri mancionem; et eciam concedendis ${ }^{20}$ per que id quomodolibet valest impediri, discrecioni tue liberam concedimus anctoritate presencium facultatem. Datum et cetora.

\section{Primum mandatum legati.}

Othobonus miseracione diuina sancti Adriani diaconus cardinalis apostolice sedis legatus de Stratford' de Bermondseye de Merton' abbatibus prioribus et conuentibus Cisterciensis Cluniacensis et sancti Angustini ordinam Iondoniensis Wintoniensis et Cantuariensis dyocesium salutem in salutis auctore. Cum ex iniuncti nobis officii debito nos opporteat ecclesiarum et ecclesiasticarum personarum statui et saluti prospicere, expedit ut que per nos ipsos circa hoc implere non possumus aliis viris discretis committamus. Qus propter super vos et ecclesias nestras summam in Christo gerentes affectum et omnis in vobis agi recte et spiritualiter et temporaliter affectantes religiosos et providos uiros fratrem Henricum de Wodestok' et consocium ordinis fratrum minorum conuentus Londonie latores presencium duximus destinando, vobis et ecclesïs uestris vice nostrs inpensuros visitacionis officium, eciam es que circs 708 invenerint fideliter nobis relaturos, $v t$ in bonis et bene placitis deo cam graciarum accione gaudere possimus; et si qua minus conneniencia uel honesta fuerint illis correccionis debite remedium apponamus. Quocircs vinersitatem uestram monemus rogamus et hortamur in domino vobis in virtute obediencie qus fungimur auctoritate mandantes quatinus

1 Printad in Regiatres de Clement IV, ed. E. Jordan, P. 14, no. 4.

13 IS. concedenda. 
prefatos fratres benigne recipientes et condigno tractantes eisdem ciras ea que pertinent ad commisse sibi visitacionis officinm obediatis humiliter et efficaciter intendstis. Alioquin sentenciss quas tulerint in rebelles ratas habebimus et faciemus suctore Doo inniolebiliter obseruari. Datum Londonie ij kal. Marcii pontificatus domini Clementis paps iij anno ij.

\section{Littere visitatoris.}

Reuerendo religionis viro domino sbbati dei gracia sancte Marie de Stratford' priorique ac ceteris tratribus vninersis Henricus de Wodestok' de ordine fratrum minoram conuentus Londonie utriusque honoris in Christo salutem et continusm sospitstem. Ex nouo ac speciali precepto domini legsti hac quints feris mihi iniuncto robis denuncio quod opportebit me ad ros accedere et auctoritate domini pape personss nestras et que circs uos geruntur et aguntur visitere. Tamen procuram uobis inducias aduentus mei usque ad feriam quartam ante dominicam in ramis palmarum. In ouius rei testimonimm ex precepto einsdem domini legati sigillum menm presentibus spposui. Datum Iondonie feris quints post dominicam qua cantatur Letare Ierusatem anno gracio Mo-CCO. seragesimo quinto.1e

\section{Acmorandum.}

t. 258\%. Anno autem domini Mo-CCo. sexagesimo quinto 16 feria quarte ante dominicam in ramis palmarum venit quidam nuncius domini episcopi Londoniensis neleius officialis noncians ouidam monacho de Stratford' in ecclesis sancti Pauli Londonie quod esdem die uenturi essent duo fratres minores ad domum suam de Stratford', missi a domino legato ut eos nisitarent. Qui videlicet fratres nenientes ad dictsm domum de S. eodem die sero obvianerunt domino abbati extra abbaciam. Volentes autem ei cansam adventus sui demonstrare, et eciam auctoritatem quam a domino legato habuerant, respondit sbbas se non pose tuno illis intendere, rogenitque eos intrare in abbaciam locuturi com priore et monachis quousque ipse rediret. Quibus ingressis et a monachis dicte domus honeste receptis scita ecism causs aduentus eorum benigne illis respondentes dicebsnt se hniusmodi visitacionem admittere non posse nec ecism debere aliquo modo marime autem in absencis abbatis sai. Nolentibus nero illis in sbbecis hospitari sed in vills miserunt illis monachi cibo et potui necessaris, dicentes se cum illis in crastino colloquinm bsbituros. Mane antem facto perrererunt ad eos ostendentes eis prinilegis sus quare haimsmadi visitacionem admittere non debebsnt, petentes ecism ab eis sibi dare indnciss quousque saltem cum abbate suo colloquinm habere possent. Illis autem dare nolentibas miserunt statim ante faciem suam duos monachos cum pritulegüis snis ad dominnm legatam. Ipsi enim dicebsnt se eorum sequi uestigis qusmcicins possent. Quo cum peruenerint dicti monschi et ibidem usque ad horam prandii morarentur nec predicti fratres ad eos uenerunt, nec ingressum ad dominum legatum habere potwerunt, sicque domi inperfecto negocio redierunt. Ferisque sutem tercis sequenti reniens ad dictam domum de Stratford' predicti domini episcopi Londoniensis officialis in propria persona, talique sccepto a domino legato mandato,
s ifs. iijj.
14 MS. poptangesima.
16 MB. septungraimo. 
citauit peremptorie videlicet dominum abbatem, priorem, cellerarium et consilium domus quod comparerent in crastino coram domino legato cum omnibus privilegiis suis et indulgenciis presens negocium contingentibus audituri quid aduersum eas esset propositurus. Comparuerunt iuxta tenorem citacionis et lectis quidem privilegiis suis respondebat ille ea nihil valere nec eins potestatem infirmari per ea in hac parte; inponens eciam eis quod predictos fratres minores ad se missos non benigne sed aspere et inhumaniter et in obprobrioss nerba prorumpentes suscipientes eos affecerunt, quod in consciencis eorum non est nec aliquis eorum vnus talin uerbs proferre posse scire potest. Sicque factum est at inducias ab eodem inpetrare non possent quousque super hac re commune ordinis sui consilinm haberent, nec eadem die alterius rei graciam consequi, set cum tali repulsa recesserunt. Statimque feria quinta sequente scripsit illis frater ille qui super eos talem a domino legato receperat potestatem, videlicet frater Henricus de Wodestok' quod ex speciali precepto domini legati iterum ueniret ad eos nisitandi gracis videlicet tali die prefigens eis diem. Interim autem dum hec agerentur miserant dicti monachi de Stratford' quosdam amicos suos ad dominam reginam supplicantes eidem ut interpellare dignaretur pro eis. Que statim sui gracis nancios suos misit ad dominnm legatum mandans ei quod pro amore suo cessaret ab inquietacionibus eorum in hac parte. Nec regine acquieuit legatus. Que tamen a precibus sic cessare nolens, mandauit alios nuncios ad eundem, ut in propria persona neniret ad se locuturus secum; quem cum multis precibus pro ista causs palsaret regina exandiri non potuit. Accesserunt ecism ad eum plures nobiles Anglie pro ista cause quorum primi erant dominus P. Basset ${ }^{18}$ et dominus R. Waletanus, ${ }^{17}$ supplicantes eidem pro illis et eciam allegantes, quorum non sunt exanditi preces, nec allegaciones allocate. Iterum autem uenerunt predictus frater et socius die quem prefixerant sero ad portas dicte abbacie quibus dicte domus monachi ingressum deneganerunt mandantes eisdem quod mane illis responderent. Illis sutem reuertentibus ad hospicinm'sunm in villa miserant monachi quod eisdem ad potum nocte ills necessarinm fuit, mane antem facto perrexerunt ad eos. Qui cum eis exponerent causam adventus sui statim monachi in prima fronte in scriptis appellauerunt, mittentes eciam eadem hora procuratores suos Londoniam qui eciam coram domino legato eandem appellacionem fecerunt. Quibus iterum ille precepit quod prior et seniores domas comparerent coram eo vigilia Pasche cum privilegiis aliis si forte plura haberent. Quod eciam factum est. Eadem siquidem die post missam suam in capella eins conspectui se presentantes minus honeste eas a se repelli fecit. Iterum autem cum intraret cameram suam steterunt ibidem petentes audienciam eins. Quo a conspectu suo repellens ut prius, precepit eis andienciam cuiusdam magistri petere qui tunc presens in curia non erat. Cui ecism scripsit sub hiis uerbis.

\section{Epistola ad commissarium.}

O. miseracione dinins a cetera discreto viro magistro G. de sancto Petro canonico Iondonie salntem in salutis auctore. Cum ex officii nostri

10 Ct. Dictionary of Natimal Biography, s.r. Sir Philip Basset.

"Ibid., s.7. Robert Walerand. 
debito super statum et reformacionem ecclesiarum quantum ad honorem dei et animarum salutem spectat secundum datam nobis a deo graciam intendentes religiosum virum fratrem $H$ He Wodestok' de ordine fratrum minorum ad monasterium beste Marie de Stratford' Cisterciensis ordinis Londoniensis diocesis misissemus, ut ibi circa quedam que in ipso mona1. 259. sterio a regularis honestatis semita declinare ad audienciam nostram peruenerat diligenter inquireret et que inueniret corrigenda corrigeret nisi talia essent que ad nos merito perferri deberent. Abbas et monachi dicti monasterii non benigne sed aspere et inhumeniter recipientes et in obprobriosa uerbs temere prorumpentes, se a nobis seu de mandato nastro visitari non posse direrunt et contra hoc se munitos apostolice sedis prinilegiis allegauerunt. Prefatus frater missus a nobis eis deferens certum diem prefixit eisdem ${ }^{\text {2s }}$ quo se coram nobis cum iuribus et defensionibus sois presentarent. Cum igitur prefsti monasterii abbas et conuentus termino sibi prefixo qui in hodiernum diem incidat minime comparuerunt coram nobis, nosque contra ipsos tanquam contra contumaces procedere possemus iuste benignius tamen et micius religionis intuitu agere cum eis cupientes, nec tamen tantum scelus silencio preterire ualentes, discrecioni tue qua fongimur auctoritate mandamus quatinus sine more dispendio ad monasterium prefatum personaliter accedens dictis abbati et conuentui peremptorie terminum prefigas vt tali die cum omnibus priuilegiis indulgenciis et iuribus suis presens negocium contingentibus per se uel per procuratorem suum ydoneum comparesnt coram nobis ex parte nostra prefato abbati necnon et priori nibilominus iniungendo vt dicts die et cetera personsliter compareant, visari et audituri que sibi doserimns proponenda. Denuncies eciam eisdem quod nisi citacioni tue paruerint, nos contra ipsos prout secundum insticiam expedire videbimus uel viderimus. Datum Londonie xiv kal. Aprilis pontificatus domini Clementis pape iij anno ij. ${ }^{19}$

Item memorandum quod hec facts sunt anno domini Mo:CCO.LXVmo 20 et quod dicti monasterii procurator apud dominum legatom nullam graciam inueniens statim lecta sollempniter eius procuracione in conspectu et andiencia multorum clericorum nidelicet et laicorum appellanit vt prius sic.

\section{Appellacio.}

Cum ego frater A. de B. commonachus et procurator religiosorum virorum abbatis et conuentus monasterii beate Marie de Stratford' Cisterciensis ordinis Londoniensis diocesis coram vobis sancto patre domino Othobono sancti Adriani diacono cardinali apostolice sedis legato alias proposuerim, me probaturum optulerim et a vobis appellauerim in forma que sequitur; coram vobis sancto patre domino $O$. et celera propono eciam ego frater A. de B. monschus monasterii de Stratford' Cisterciensis ordinis Iondoniensis diocesis procurator abbatis et conuentus eiusdem monasterï procuratorio nomine pro eisdem abbste et conuentu. Quia cum a sede apostolica abbati Cistercii einsque coabbatibus et conuentibus sit concessum vt a nullo nisi a patribus abbatibus sen eiusdem ordinis monachis a dictis patribus abbatibus super hoc deputatis visitari uel corrigi
" IIS. eidem.
1. MS. iiij.
$=$ MS. $H^{0} C^{\circ} \operatorname{LxX}=0$. 
ualeant, ${ }^{21}$ sitque concessio memorata per statutum sedis eiusdem nihilominus roborata, quod me offero nomine et vice dictornm abbatis et conuentus pro loco et tempore coram indice competenti legitime probaturam, vos pie pater volentes in prefato monasterio per vos uel alium uel per alios visitacionis et correccionis officium exercere, salus in omnibus et per omnia uestre sancte paternitatis reuerencia, dico quod hoc facere non potestis, nec de iure debetis ex officio legacionis generaliter vobis commisse. Sane licet eadem anctoritate sit decretum irritum et insne si contra concessionem supradictam aut ststutum memoratum a quoquam fuerit presumptum, sitque decretum quod si alique sentencie in abbstes et conuentus $\$$ supradictos occasione huinsmodi fuerint prolate, nullam robur optineant firmitatis ; sit eciam concessum eisdem a sede apostolica memorata ne aliquis legatus sedis einsdem sine speciali mandato dicte sedis in eosdem abbatem et conuentum predictos aut in eorundem monasteriis aliquas excommunicacionis suspensionis nel interdicti sentenciss contre ea que ipsis a dicta sede indulta sunt promulget." Tamen ego procurator prefatus metuens ne si vos sancte pater per vos uel alium uel alios uisitacionis officium in preiudicinm concessionis prefste et statuti supradicti exercere velitis in monasterio supradicto eciam contingeret abbatem et conuentum supradictos vos uel alium seu alios uestro nomine ad hoc non admittere, ne propterea aliquam seu aliquas interdicti suspensionis aut excommunicacionis sentenciam seu sentencias in prefatam abbatem seu aliquem vel aliquos de dicto conuentu vel in ipsum conuentum aut in monasterium prefatum, de facto per vos uel per alium uel per alios proferatis uel proferri mandetis, nomine et vice supradictorum sbbstis et conuentus sanctam sedem apostolicsm in hiis scriptis appello et appellaciones instanter peto, supponens supradictos abbatem et conuentum necnon et eorum monasterium et ecclesiam et statum ipsorum ac eciam concessionem prefatam et statutum memoratum et alia priuilegis eisdem et aliis de eorum ordine a sede apostolica concessa protectioni et defensioni sedis apostolice memorate. Item ne aliter uel alio modo sbbatem et conuentum predictos uel eorum monasterium aut ecclesiam grauetis, seu contra priulegis eisdem a sede apostolica indulta aliquid per vos uel alium seu alios attemptetis, sedem spostolicam nomine et vice dictorum abbatis et conuentus in hïs scriptis appello et appellaciones instanter peto. Et cum vos, sancte pater, post hoc preceperitis quod prior et, officiales supradicti monasterii uestro se conspectui certa die presentarent ostensuri concessionem statutum et privilegis sedis apostolice, de quibus in saperioribus habetur et fit mencio et sic comparuerint, et de hiis uestre sancte paternitati inde fecerint plenam fidem, appellaciones f. $250^{\circ}$. supradictas alias uel alia vice interpositas a vobis procuratoris nomine pro abbste et conuenta supradictis innouo, et iterum ut prius propono et appello coram vobis sancto patre domino 0 . sancti Adriani diacono et cetera ut supra. Item ne aliter uel alio modo abbatem et conuentum predictos et cetera ut superius notatum est.

a Regula, Constitutiones, ef Privilegia Ordinis Cistercicnsis, cd. Henriquez, p. UH, no. xI; p. 68, no. xrri. 7 ISS. conuentos.

- Regala, Constitutiones, et Privilegin Ordinis Cisterciensis, P. 59, no. xi; p. 73, no. Ixrvii. 


\section{Liberaciones.}

Item memorandum quod iste legatus inhibuit ubi visita uit, scilicet in nigro ordine, fieri liberaciones secularibus ${ }^{2}$ que solebant concedi in hac forma. Viniersis sancte matris ecclesie et cetera frater $P$. dictus uel vocatus prior de tali loco et eiusdem domus conuentus salutem in domino sempiternsm. Noveritis nos vnenimi assensu et pari uoluntate dedisse concessisse et hac presenti carts nostra confirmasse tali, scilicet aliqus persona nominats, solo caritatis intuitu cum vao garcione et vno equo in domo nostra sustentamentum suum et honestum hospicium cum sufficienti focali in suo perpetuo uel quosd nixerit nel ad surm vitam, videlicet tot panes in die uel in ebdomada sibi de pane conuentuali et tot lagenas uel galones cernisie connenturlis, et diebus qui comedunt carnes tot fercula competencis quoram duoram generam uidelicet vnum de carne salsa seu sallita et aliud de insulsa vel tot bacones per annum et tot carcosia uel corpora boum et tot multones, et diebus quadragesimalibus et quibus commeduntur pisces tot fercula piscium competencis et que ipse durerit acceptare; et nibilominus diebus piscinm quibus potest lacteus cibus nel ous commedere racionabilem quantitatem casei et butiri vel tot petras per annum; et ad servientem suum de pane grossiori et cernisia seruiencium tantum vel sic vni puerorum qui sunt in stabulo prioris; et ad equam sunm fenum et prebendam sicut palefrido prioris uel tantum, et in estiuo tempore quando equi herbam commedunt herbagium competenter et sufficienter inueniemus; habendum et percipiendum in domo nostra omni tempore uite sue sine contradiccione cuinscumque. Ista autem omnis eidem $\mathrm{N}$. in suo perpetuo contra omnes fideliter warantizsbimus et solo caritatis intuita persolnemus. Si nero alibi morari noluit nihilominus predictam liberacionem per nuncinm suum quemcumque mittere uoluerit percipiet; et utrum noluerit semel in ebdomada pro tota septimana percipere nel

- MS. 9. cotidie sicut conuentus, in sua uoluntate esset. Et ut hec * nostra donacio a cetera pro nobis et successoribus nostris huic scripto sigillnm nostrum apposuimus. Ista antem plenarie faciemus sub pena decem solidorum operi maioris ecclesie de $\mathrm{N}$. solaendoram quocienscumque aliquod horum omisimus uel in liberacione tardanerimus, hiis testibus et cetera.

Item aliter. Noueritis nos solo caritatis intuita concessisse tali omni tempore vite sue ad sustentamentum suum illud et illad a nobis annuatim ad tales terminos ibi aliquo loco nominato percipiendum, videlicet ad illum terminum hoc et ad illum illad. Si autem aliquo tempore ei propter precipusm sollempnitatem uel manifestam corporis sui inflmitatem ei uberins uel curialius pronisum uel ministratum fuerit, non poterit hoc in consuetudinem trahere uel a nobis hoc exigere nel extorquere at cetera.

Fel Noueritis nos teneri tsli in tanto a nobis solo caritatis intuita concesso et percipiendo in tali loco quousque eidem de competenti ecclesiastico beneficio quod quidem ipse durerit acceptsndum proniderimns uel per nos pronisum fuerit et cetera.

Item. Noneritis nos teneri domino $\mathrm{N}$. de Le militi pro aurilio et seruicio suo nobis et hominibus nostris in illis duobus comitstibus abique et' sine ficcione cum tamen premunitus fuerit de negocio impendendo in

24 This was farbidden in the Constitutions of Ottoboni in 1288: Wilkins, Concilia, ii, p. 17, cap. zlviii. 
tantum persoluendum eidem et $a b$ eo uel speciali attornato suo ad hoc deputato et directo percipiendum ad illas nundinas annustim quousque circulus octo annorum plene compleatur. Ad quod faciendam obligamus nos et domum nostram districcioni et cohercioni illius balliui concedentes quod possit nos per bons nostra in ballius sua existencis de die in diem compellere quousque dicto domino $\mathrm{N}$. competenter satisfecerimus. Et si testes idoneos habere potuerimus ad probandum quod ipse seruicium suum uel aurilium a nobis uel nostris postulatum denegauerit licebit nobis dictum redditum eciam ante terminum $a b$ eo uel ei subtrahere uel retinere. In cuins rei testimonium vel ad maiorem securitatem huic scripto et cetera.

Procuratorium ad mutuum contrahendum muturum ${ }^{20}$ quasi in eum terminum.

Vninersis et cetera abbas de Stratford' Cisterciensis ordinis Londoniensis diocesis et einsdem loci humilis conuentus in domino salutem eternsm. Mittimus dilectos nostros in Christo flios fratres A. et B. priorem et cellerarium domus nostre latores presencium ad nundinas sancti Botulphi, (Vel noneritis quod nos constituimus, facimus et ordinamus illum et illum commonachos nostros) speciales procuratores et attornatos nostros ad matanm contrahendum cam quocumque fideli seu cum quibuscumque fidelibus de C. libris argenti ad prouisiones necessarias domus nostre faciendas et procurandas cum ad presens nos grauia et exquisita ad hoc faciendum urgeant negocia. Obligamus eciam nos et domum nostram et omnia bons nostra mobilia et immobilia ecclesiastica et mundana ubj- t. 280. cumque seu quibuscumque locis existencia creditori nostro seu creditoribus nostris quibuscumque aput quem uel aput quos graciam negocii huiusmodi expediendi inuenerint, scilicet a quo uel a quibus pecuniam prenominatam mutuo acceperint, ad omnem illam pecuniam fideliter et sine ulteriori retencione dilacione uel dolo persoluenda die et loco seu diebus et locis inter eosdem procuratores uidelicet nostros predictos et creditorem nostrum seu creditores nostros ai plures fuerint constitutis ratam stabilem et gratam habituri conuencionem quamcumque uel qualemcumque prenominati procuratores nostri cum quocumque creditore uel cum quibuscumque creditoribus in scriptis confecerint. In cuins rei testimoninm presentes litteras sigillo nostro maiori et communi signatas ad omnimodam securitatem per predictos procuratores et atornatos nostros creditori nostro ael creditoribus nostris transmittimus patenter. Valete in domino semper. Datum et cetera.

Nata quod in procuratoriis nunquam bene ponitur preteritum tempus uel preteritum plusquam perfoctum, verbi gracia, Noueritis quod nos constitnimus fecimus et cetera, uel Noveritis nos constituisse et cetera, propter disputaciones que tunc insurgunt inter causidicos uel logistas.

\section{Aliud genus.}

Item aliud ad mutusem contrahendum. In omnibus causis et negociis nos domum uel ecclesiam nostram maxime ad instantes nundinas sancti Botulphi qualitercumque tangentibus dilectos. filios et commonschos nostros et cetera constituimus facimus et ordinamus, dando eisdem plensm potestatem agendi defendendi excipiendi replicandi appellandi tot saccos bone lane per decem [annos] de rebus nostris ubique ex parte

as 3is. in his. :a 31 S. repeats mataum. 
nostra pre manibus disponendi vendendi et pecuniam pro dicte lane uendicione pre manibas percipiendi ecclesias nostras de A. et de B. per decem annos ponendas ad firmsm sub quacnmque conuencione nobis niderint expedire; domnm nostrum et omnia nostra quibuscumque et sub quacumque forma uerborum uel quocumque modo nobis viderint expedire obligandi, ac eciam omnia alis faciendi et dicendi quecumque nos si presentes essemus facere possemus aut dicere, et quecumque ueri et legitimi procuratores facere poterunt aut debebunt. In cuins rei testimonium et cetera. Valete et cetera. Datum et cetera.

\section{Obligacio.27}

Vninersis et cetera [abbas] de Stratford' et eiusdem domus conuentus Cisterciensis ordinis Londoniensis diocesis at cotera. Noueritis vniuersitas uestra nos teneri et hoc scripto obligatos esse A. filio B. cini de Londonia in viginti libras sterlingorum bone integre et legalis monete legaliter numeratorum quas ab eodem in magnis necessitatibas et pro grauibus et arduis negociis que nos tunc urgebsnt monasterï nostri et vtilitatibns ecclesie domns nostre ntiliter expediendis dominica quarta post pascha anno domini Mo et cetera aput Londoniam mutuo accepimus. De cuius pecunie solucione eidem nel atornsto suo specisliter ad hoc deputato et nobis hoc scriptum deferenti sine restituenti tali die anno proximo venturo absque omni dolo fraude vel ulterion ${ }^{*}$ retencione uel diuturniori dilacione plenarie integre et fideliter aput dictam civitatem de L. satisfaciemus sub pena decem solidorum tali archidiacono uel tali indici uel ballino uel operi tslis ecclesie soluendorum. Si nos quod absit in dicte pecunie solncione contigerit dictis die locoque defecisse nel si in parte uel in toto defecerimus rel quam quidem pecuniam eidem persoluemus et cetera ut supra vel si solucionem tardanerimus uel pacscionem distulerimus. Et ad hec omnia legitime sicut supradictum est faciends obligamus nos et omnia bona nostra ecclesiastica et mundana iurisdiccioni potestati coherccioni et districcioni vel subicimus nos at cetera talis archidiaconi nel talis balliui qui pro tempore fuerit; concedendo uel concedentes quod possit nos per predictam pensm uel qualitercumque noluerit de die in diem uel incessanter compellere et distringere quousque dicto $A$. de dicte pecunie solncione competenter ut dictum est satisfecerimus. Fxpensas autem, si quas miserit aut fecerit, expectando pacacionem snam ultra statutum diem eidem uel atornato suo allocabimus et refundemus. Renunciamus ecism in premissis ael renunciantes et cetera omni escepcioni cavillacioni regie prohibicioni omnibus litteris sen privilegiis sen indulgenciis inpetrstis et inpetrandis et omni iuris aurilio uel remedio canonici et ciuilis, quod uel que in hac parte nobis prodesse et sibi obesse posset nel passent. In cuius rei robur et testimoniom eciam at enndem securum redderemus hoo scriptam sigillo nostro communi signstas eidem litteras fecimus fieri patentes. Valete et cetera. Datum.

Pel Noveritis nos recepisse et habuisse aput Iondoniam in pecunia

7 In 1286 the Abbot of Stratfard borrowed moner from Inondon Jews: Cal. of Pat. Rolls, 2258-66, p. 566; 26id. 2206-72, p. 496. At the roqueet of his mother, Queen Elesaor, Edward I scquitted the Abbot and convent of Stratford of wroties on all debts due to the Jews, ssuing to the Jews their principal debts: ct. Cal.of Clace Rolls, 2078-9, P. 140. is. IIS. viterione. 
numerata tali die illius anni ab illo et illo mercatore soluentibus tam pro se quam pro illo et illo sociis suis ciuibus et mercatoribus Florentinis, uel Florentibus, tot libras sub tanta pecunia eisdem soluendis tali anno et f. $260^{\circ}$. tali die si ob regiam inhibicionem aut acta de lane nostre uel decimarum de tali loco uendicione conuencio in suo statu et robore stare et permanere non potuerit. Si autem supradicta conuencio regali inhibicione non obstante nec inpediente firmiter usque ad statutum tempus in antedicta conuencione et stabiliter perseuerauerit et durauerit, predicte tot libre dictis mercatoribus in pacacione sua pro dicta lana seu pro dictis decimis a nobis sine condicione allocabuntur. Ad quod fideliter faciendum obligamus et subicimus nos et omnia bons nostra ubicumque existencia dictis mercatoribus; concedentes quod possint nos secundum leges et consuetudines mercatorum ad nundinas sancti Botulphi distringi facere uel distringere si eisdem at dictum est dictam pecuniam si tamen soluenda fuerit fideliter non persoluerimus, vel si allocanda fuerit, eam non allocsuerimus. In cuius rei testimonium et cetera. Valete et cetera. Datum et cetera.

$\nabla$ el Noueritis nos anno domini et cetera tali die mutuo recepisse ab tali tantam pecuniam, de qua pecunia uel de cuius pecunie solucione vel quamquidem pecuniam at cetera pro nobis et successoribus nostris apposuimus. Et hec est forma probabilis et usualis multum faciendi obligaciones uel in dacione obligacionum.

\section{Peticio consilii.}

Magne discrecionis viro et amico suo specialissimo et confidentissimo magistro $N$. rectori ecclesie de $N$. sui semper denotí fratres $R$. dictus abbas de Stratford' et eiusdem loci humilis conuentus salutem, et sic transire per tempora ut non amittat eterna. Licet per uestram prudenciam et mirificam sapienciam quedam negocia nostra grauia expedita sint et ad effectum debitum deducta et mancipata, tamen adhuc restant expedienda vobis 2 feliciter et ad domini beneplacitum grauiora. Vndique enim aduersarii et inimici nostri coñsurgentes vallo persecucionum et placitorum nos circumdederunt, ita quod continua nobis foris inest pugna et innumeri intus manent timores. Ecce enim ille antiquus aduersarius noster videlicet ille magnas qui nos per tale placitum inquietare et verare per modum accionis uel in curia domini regis solebat de nono prius occultas resumpsit uires, et illud breue quod olim expirauerat per mortem predecessoris sui iam leuauit et resuscitauit vtique quo dicior eo forcior ad nocendum. Omnes enim hiis diebus diligunt muners ${ }^{30}$ et cetera. Insuper et ille dominus legatus nos adhuc fatigare non cessat immo eciam libertates et indulgencias ordini nostro concessas et hucusque in ordine usitatas et approbatas stipatus utique consilio et instigacione multorum prelatoram secum existencium pro viribus nititur adnilare inpugnare et infirmare. $\mathrm{Ob}$ quam rem tali die ab eo ad sanctam sedem Romansm appellauimus in forma iuris. Et propter hoc et alia multa que nimis esset longum enarrare et per singula enumerare, in presenciarum in tam arto positi sumus quod necessitate legem non habente compellimur uel com-

$\Rightarrow$ IIS. $\nabla \bar{t}$.

20 Isaish i. 23. The JIS. has in mikericordiu, the scribe having apparently misread mun'a $2 s$ in mia. 
puli[mur] amicos nostros vniuersos et singulos omnes ad consilinum nostrumque ubicumque existentes conuocare, uestram discrecionem et cetera.

Del Quum inter nos et talem nuper, uel de nouo, nowa lis seu contencio orta est que sine magnorum consilio et discrecione finem debitum sortiri non potest, vade et ad esm sine uestro consilio et disposicione manum apponere non audemus, pro qua quidem lite hac instanti die iouis apud Cestr' coram domino archidiacono comparere debemus, nescientes adhuc quid ipse agere uel proponere intenderit, nec habemus ad presens alicuius alterius tatom et securam consilium cui nos contra dicti aduersarii nostri insidias et maliciam, qui pro viribus nos exheredare disponit et intendit, committamus, discrecioni uestre attente supplicamus et deuote quatinas omni occasione remota ceteris interim pretermissis, licet nobis grane sit et sumptuosum quod bene nouimus sic commodum et honorem nostrum diligitis hac instanti die Lnne super dicto negocio tracteturi, et nobis super hoc consilii nestri et aurilii impensuri beneficium; ad nos uel ad domum nostram personsliter accedatis, nel nobis presencism uestram exhibere seu presentare nelitis; $\nabla t$ per uestrum consilinm et per uestram prodencism dicti aduersarii nostri maliciam, qui nos nexare non cessat, caucius euitare possimus. Tantum igitur si placet faciatis ne domus nostra contra priailegiorum nostrorum indulgencias in aliquo casuram paciatur vel ad aliquam deücionem seu apporiacionem, quod ad vestre discrecionis lesionem cederet quod absit, cum et vos ita cum ipso sitis ut nos tamen non deseratis deueniat, uel aliquod dampnum sustineat in presenti.

Vel Qunm in necessitatis articulo solebsnt amici specialiores et discreciores requiri, in quorum discrecione uel audacia maior pendet fidncis, ad $70 s$ tanquam ad vnicum et singulare in hac parte refuginm necessitate ducti recurrimus, rogantes attencius quatinus super magnis et arduis negociis libertates ecclesie nostre tangentibus tractaturi tali die ad nos et cetera.

Vel Noueritis nos a quibusdam parochisnis nostris uel parochianorum nostroirum inplacitari de quibusdam decimis que forsitsn dari non consueueront quas nos violenter asportsuimus; pro quo placito ad prosequendum querelam nostram ibi tali die coram tali comparere debemus. Propterea vos rogamus rel quapropter vel ob quam rem et cetera. Tantum igitur pro nobis in absencia capitis uel prelati nostri si placet faciatis quod ipse qui nuper uersus Londoniam iter arripuit cum aduenerit preter certsm et debitam conduccionem et mercedem merito vobis teneatur ad grates uel graciarum acciones et ad obsequia.

\section{Dispersacio.}

Viro reuerentissimo sumai pontificis vicario domino 0 . miseracione diuina sancti Adriani diacono cardinali apostolice sedis legato suns si placet filius deuotus frater N. dictus abbes de Stratford' Cisterciensis ordinis Iondoniensis diocesis utriusque honoris in Christo salatem et pedum ascula beatorum. Firmiter credimus et confitemur quod in

f. 261. aduentu uestro ad regnum Anglie visitauerit nos oriens ex alto. Qui uenistis utique ad consolendum pusillanimes et medendum contritis corde ac pauperibus euangelizandum. Hinc est pater reuerende quod latores presenciom fratres A. et B. commonachos nostros vestre destinamus 
clemencie, deprecantes et excusantes in domino quatinus si dispensacionis locus est in casibus eorum scilicet casus in transcriptis vobis inspiciendis non notarï manu sed nostrs scriptis et sub sigillo nostro vestre paternitati transmissis cum eisdem si placet dispensare uelitis. Verum quidem et fidele testimoniom perhibentes eisdem dicimus in veritate quod satis contristantur super errore suo oitam honestam cum conuentu fratrum suoram ducentes et pacificam, sed nt eo feruencius sub disciplins regulari militare ualesnt quo propinquins dininis fuerint coniuncti petinerint pro deo ab altaris ministerio diucius non separari. Hnius antem rei cause beneuolencie uestre complementum assiduis exoramus anxietatibus clamoribusque humilibus homiliter supplicantes quatinus super aftictos pia gestantes viscera prouida consideracione condescendatis, casibus horum fratrom nostrorum si aliqua indalgencis possint in gradu sacerdotali dininum officium exequi et exercere. Periculosum enim eis esset ad dominum papam Rome existentem transalpinare et per tot galaxias incedere deficientibus expensis ob nimism panpertatem. Mandatum uero beneplaciti uestri expectamus languentes filiorum nostrorum egritudine, sed Salomone dicente quis legatus fidelis, sanitas, 31 non desperamus sauciati, si uobis placuerit uestra consuets benignitas 2 que ubique puplice circumdatar et prinatim dolorem nostrum temporare et mitigare. Conseruet altissimus incolumitatem uestram ecclesie sue per tempora longa. Datum et cetera.

\title{
Memoranda of Hugo de Assendelff and others
}

Iv the library of Corpus Christi College, Oxford (MS. 462, A. 5. 20) is a copy of the Utrecht missal printed in Paris by John Higman for Wolfgang Hopyl, 30 November 1497. Being on vellum the book is suited for the preservation of family records; and for this purpose it was used by a member of the family of Assendelff, or Assendelft, which took its name from a village and estates lying about ten miles to the NE. of Hasarlem. On the verso of $f$. 1 before the title a name is inscribed, with marks of possession; but subsequently, when the book passed, perhaps by presentation, to Master Hugo de Assendelff, the first owner's name was effectually erased, and all that can now be read is that he was 'wonende toe Delft'. The calendar prefixed to the missal is full of manuscript notes in various hands, which can without difficulty be identified. They chronicle a few public events ; but, as is natural, are concerned mostly with the family, recording births, marriages, and deaths of its members.

Master Hugo was born on 3 November 1467. As a boy he saw the enthronement of Maximilian at Harlem in March 1478; and was present when his great-uncle, Hugo, founder of the Cistercian house at Heemstede, died in February 1483. In 1487 he was M.A. at Louvain, and ten years later became Licentiate

\author{
3 Proverbs miii. 17.
}

n MS. benigon.

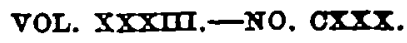

